\section{OP0265 VALIDATION OF A DEFINITION FOR ATTACK (FLARE) IN PATIENTS WITH ESTABLISHED GOUT}

A. Gaffo ${ }^{1,2}$, N. Dalbeth ${ }^{3}$, J. Singh ${ }^{1,2}$, K. Saag ${ }^{1}$, W. Taylor ${ }^{4} .{ }^{1}$ University of Alabama at Birmingham; ${ }^{2}$ Birmingham VA Medical Center, Birmingham, United States; ${ }^{3}$ University of Auckland, Auckland; ${ }^{4}$ University of Otago, Otago, New Zealand

Background: A standardized validated definition for gout attacks (flares) is not available. Two provisional definitions published in 2012 were based on patientreported elements (patient-defined attack, pain at rest greater than 3 in a 0-10 numeric rating scale, presence of at least one swollen joint, presence of at least one warm joint) (1). These definitions had acceptable sensitivity and specificity but lacked external validation which is necessary before they can be adopted in gout clinical studies.

Objectives: To perform external validation of previously published preliminary gout attack (flare) definitions in patients with gout.

Methods: We enrolled 509 participants with gout from 17 international sites in a cross-sectional study performed during routine clinical care. All patients met the 2015 ACR/EULAR classification criteria for gout (2). Criteria for the previously published gout attack definitions were collected by a site investigator and the final adjudication of a gout attack status was done by a local expert rheumatologist, through an evaluation independent from that of the site investigator. Logistic regression, Bayesian statistics, and receiver-operator curves were used to calculate the final diagnostic performance of the gout attack definitions.

Results: The mean age of participants was 57.5 years (standard deviation [SD] 13.9 ) and $89 \%$ were men. Mean disease duration was 12.3 (SD 10.3) years, $35 \%$ had tophi, and $75 \%$ were taking urate-lowering therapies. The previously published and favored definition requiring the presence of 3 or more out of 4 criteria ("number of criteria") was found, using the current study data, to be $85 \%$ sensitive and $95 \%$ specific in confirming the presence of an attack in patients with gout (Table). The concurrent logistic regression model had an area under the curve of 0.97 . The previously published definition based on a classification and regression tree algorithm (entry point pain at rest $>3$ followed by patient-defined attack "yes") was $73 \%$ sensitive and $96 \%$ specific using the current study data (Table). The "number of criteria" approach with a cut-point at 3 or more out of 4 criteria had higher diagnostic accuracy using the current study data than in its initial 2012 description (92\% versus $84 \%$, table). (1) Finally, using current study data the "number of criteria" approach at 3 or more out of 4 criteria had higher accuracy to the classification and regression tree algorithm based approach (92\% versus $89 \%$ ) but with a much better sensitivity ( $85 \%$ versus $73 \%)$.

Table. Diagnostic performance of gout attack (flare) definitions: "number of criteria"* and classification and regression tree (CART)

\begin{tabular}{|l|c|c|c|c|c|}
\hline & $\begin{array}{c}\text { Sensitivity\% } \\
(95 \% \mathrm{Cl})\end{array}$ & $\begin{array}{c}\text { Specificity\% } \\
(95 \% \mathrm{Cl})\end{array}$ & $\begin{array}{c}\text { PPV\% } \\
(95 \% \mathrm{Cl})\end{array}$ & $\begin{array}{c}\text { NPV\% } \\
(95 \% \mathrm{Cl})\end{array}$ & $\begin{array}{c}\text { Accuracy\% } \\
(95 \% \mathrm{Cl})\end{array}$ \\
\hline Number of criteria (current study) & & & & & \\
\hline 1 or more & $100(100)$ & $66(61-715)$ & $57(51-63)$ & $100(100)$ & 77 \\
\hline 2or more & $97(94-100)$ & $88(84-91)$ & $78(72-84)$ & $98(97-100)$ & 91 \\
\hline 3 or more & $85(80-91)$ & $95(93-97)$ & $88(83-93)$ & $94(91-96)$ & 92 \\
\hline All 4 & $61(54-69)$ & $98(97-100)$ & $94(90-99)$ & $85(82-88)$ & 87 \\
\hline $\begin{array}{l}3 \text { or more (2012 provisional } \\
\text { definition) }\end{array}$ & $91(80-97)$ & $82(75-88)$ & $64(52-74)$ & $96(91-99)$ & 84 \\
\hline CART (current study) & $73(66-80)$ & $96(94-98)$ & $90(84-95)$ & $89(86-92)$ & 89 \\
\hline CART
\end{tabular}

$\begin{array}{llllll}\text { CART (2012 provisional definition) } & 83 & 90 & 85 & 91\end{array}$

$C l=$ confdence interval, $P P V=$ positive pre

CART = classification and regression tree

*Criteria include: patient-defined gout attack, pain at rest greater than 3 in an 0-10 numeric rating scale,

Presence of at least one swollen joint, presence of at least one warm joint

+3 or more criteria was found to have the best performance in a receiver-operator curve analysis

fCART rule: Pain at rest $>3$ followed by patient-defined gout attack positive

Conclusions: The definition requiring the presence of 3 or more out of 4 patientreported criteria is validated to be sensitive, specific, and accurate in identifying attacks (flares) in patients with gout using an independent large international sample. Having a validated gout attack definition will improve ascertainment of outcomes in gout clinical studies.

References:

[1] Gaffo AL et al. Arthritis Rheum. 2012;65:1508.

[2] Neogi T et al. Ann Rheum Dis 2016;75:473.

Acknowledgements: Funding provided by AstraZeneca, Ardea Biosciences, Inc., and Ironwood Pharmaceuticals.

Disclosure of Interest: A. Gaffo Consultant for: SOBI, N. Dalbeth Grant/research support from: AstraZeneca, Consultant for: Takeda, Pfizer, AstraZeneca, Cymabay, and Crealta, Paid instructor for: Takeda, AstraZeneca, J. Singh Grant/research support from: Takeda, Savient, Consultant for: Savient, Takeda, Regenron, Merz, Bioiberica, Crealta, Allergan, WebMD, UBM LLC, American College of Rheumatology, K. Saag Consultant for: Takeda, Horizon, SOBI, W. Taylor Consultant for: Pfizer, AstraZeneca, Abbvie, Roche

DOI: 10.1136/annrheumdis-2017-eular.3219

\section{OP0266 PRESENCE OF MONOSODIUM URATE CRYSTALS BY DUAL-ENERGY COMPUTED TOMOGRAPHY IN GOUT PATIENTS TREATED WITH ALLOPURINOL}

N. Dalbeth ${ }^{1}$, S. Nicolaou ${ }^{2}$, S. Baumgartner ${ }^{3}$, J. Hu ${ }^{3}$, M. Fung ${ }^{3}$, H.K. Choi ${ }^{4}$ ${ }^{1}$ University of Auckland, Auckland, New Zealand; ${ }^{2}$ Vancouver General Hospital and University of British Columbia, Vancouver, Canada; ${ }^{3}$ Formerly Ardea Biosciences, Inc, San Diego; ${ }^{4}$ Harvard Medical School and Massachusetts General Hospital, Boston, United States

Background: Chronic hyperuricemia predisposes to deposition of monosodium urate (MSU) crystals in musculoskeletal and other tissues, causing chronic inflammation, acute gout flares, joint damage, and disfiguring tophi. Dual-energy computed tomography (DECT) is a useful imaging tool to detect and quantify MSU crystal deposits.

Objectives: This study assessed the evidence of MSU crystal deposition using DECT scanning among gout patients treated with allopurinol and the potential determinants associated with the observed deposits.

Methods: The multicenter DECT study recruited patients with gout from the USA and New Zealand who were taking allopurinol at $\geq 300 \mathrm{mg}$ daily for at least 3 months. MSU crystal deposition was measured using DECT in hands/wrists, knees, and feet/ankles bilaterally. The presence of MSU crystals as well as the total volume of crystals were assessed according to gout characteristics and serum uric acid (sUA) levels.

Results: Patients $(\mathrm{N}=153)$ were predominately male $(92.2 \%)$, with mean (SD) age 58.5 (11.4) years, and gout duration 14.9 (10.3) years. sUA was $\geq 6.0 \mathrm{mg} / \mathrm{dL}$ in $49.0 \%$ of patients. $81.7 \%$ of patients took allopurinol at a stable dose of $300 \mathrm{mg} /$ day and the remainder at $>300 \mathrm{mg} /$ day. $69.1 \%$ of patients had MSU crystal deposits with a total median crystal volume of $0.16 \mathrm{~cm}^{3}$ (range, 0.01 to $19.53 \mathrm{~cm}^{3}$ ). Those with $\mathrm{s} \cup \mathrm{A}>6.0 \mathrm{mg} / \mathrm{dL}$ and palpable tophi showed the highest prevalence of urate deposits (90\%), and those with sUA $<6.0 \mathrm{mg} / \mathrm{dL}$ and no palpable tophi showed the lowest prevalence (47\%). Those who reported a gout flare within the prior 3 months (versus none), were prescribed allopurinol doses $>300 \mathrm{mg}$ (versus $300 \mathrm{mg}$ ), and had palpable tophi (versus none) had greater deposit volume.

\begin{tabular}{|c|c|c|c|c|c|}
\hline & \multicolumn{2}{|l|}{ Tophi } & \multicolumn{2}{|l|}{ No tophi } & \multirow{2}{*}{$\begin{array}{l}\text { Total } \\
(\mathrm{N}=153)^{2}\end{array}$} \\
\hline & $\begin{array}{l}\text { sUA } 26.0 \\
\mathrm{mg} / \mathrm{dL} \\
(\mathrm{n}=20)\end{array}$ & $\begin{array}{l}\mathrm{sUA}<6.0 \\
\mathrm{mg} / \mathrm{dL} \\
(\mathrm{n}=28)\end{array}$ & $\begin{array}{l}\mathrm{SUA} \geq 6.0 \\
\mathrm{mg} / \mathrm{dL} \\
(\mathrm{n}=55)\end{array}$ & $\begin{array}{l}\mathrm{SUA}<6.0 \\
\mathrm{mg} / \mathrm{dL} \\
(\mathrm{n}=50)^{2}\end{array}$ & \\
\hline $\begin{array}{l}\text { Median volume (range) for } \\
\text { nonmissing urate deposits }\end{array}$ & $\begin{array}{l}0.26 \\
(0.00,19.53)\end{array}$ & $\begin{array}{l}0.16 \\
(0.00,4.63)\end{array}$ & $\begin{array}{l}0.09 \\
(0.00,1.23)\end{array}$ & $\begin{array}{l}0.00 \\
(0.00,0.89)\end{array}$ & $\begin{array}{l}0.07 \\
(0.00,19.53)\end{array}$ \\
\hline \multirow{3}{*}{$\begin{array}{l}\text { Presence of urate deposits, } \\
\mathbf{n}(\%)\end{array}$} & $\begin{array}{l}18 / 20 \\
(90.0)\end{array}$ & $\begin{array}{c}20 / 28 \\
(71.4)\end{array}$ & $\begin{array}{l}44 / 55 \\
(80.0)\end{array}$ & $\begin{array}{l}23 / 49 \\
(46.9) \\
\end{array}$ & $\begin{array}{l}105 / 152 \\
(69.1) \\
\end{array}$ \\
\hline & \multicolumn{2}{|c|}{$P=0.16^{6}$} & \multicolumn{2}{|c|}{$P<0.001^{\circ}$} & \\
\hline & \multicolumn{4}{|c|}{$P<0.001^{\circ}$} & \\
\hline $\begin{array}{l}\text { Median volume (range) for } \\
\text { positive scans }\end{array}$ & $\begin{array}{l}0.37 \\
(0.01,19.53)\end{array}$ & $\begin{array}{l}0.29 \\
(0.05,4.63)\end{array}$ & \begin{tabular}{|l|}
0.12 \\
$(0.01,1.23)$
\end{tabular} & \begin{tabular}{|l|}
0.14 \\
$(0.01,0.89)$
\end{tabular} & \begin{tabular}{|l|}
0.16 \\
$(0.01,19.53)$
\end{tabular} \\
\hline
\end{tabular}

Conclusions: Despite a stable dose of allopurinol for more than 3 months, and even with sUA at the target level, a substantial proportion of patients with gout continue to have evidence of MSU crystal deposition by DECT scan. Patients with palpable tophi, sUA levels $>6.0 \mathrm{mg} / \mathrm{dL}$, and gout flares within the prior 3 months have a greater volume of MSU crystal deposition. These patients may need continuation and/or intensification of their urate-lowering therapy regimen. Acknowledgements: This study was sponsored by Ardea Biosciences/AstraZeneca.

Disclosure of Interest: N. Dalbeth Grant/research support from: AstraZeneca, Consultant for: AstraZeneca, Fonterra, Takeda, Pfizer, Cymabay, Crealta, Speakers bureau: Menarini, AstraZeneca, Takeda, S. Nicolaou Grant/research support from: Siemens Healthcare, S. Baumgartner Employee of: Ardea Biosciences, Inc, J. Hu Employee of: Ardea Biosciences, Inc, M. Fung Employee of: Ardea Biosciences, Inc, H. Choi: None declared

DOI: 10.1136/annrheumdis-2017-eular.3666

\section{OP0267 SERUM URATE AS A SURROGATE ENDPOINT FOR GOUT FLARES: RESULTS OF A SYSTEMATIC REVIEW AND META-REGRESSION ANALYSIS OF RANDOMIZED TRIALS}

L. Stamp $^{1}$, M. Morillon ${ }^{2}$, W. Taylor ${ }^{3}$, N. Dalbeth ${ }^{4}$, J. Singh $^{5}$, R. Christensen ${ }^{6}$.

${ }^{1}$ Medicine, University of Otago, CHRistchurch, Christchurch, New Zealand;

${ }^{2}$ Odense University Hospital, Odense, Denmark; ${ }^{3}$ University of Otago,

Wellington; ${ }^{4}$ University of Auckland, Auckland, New Zealand; ${ }^{5}$ University of Alabama, Birmingham, United States; ${ }^{6}$ Musculoskeletal Statistics Unit, The Parker Institute, Odense, Denmark

Background: The primary outcome measure for efficacy in clinical trials of urate lowering therapy (ULT) is frequently serum urate (SU), effectively acting as a surrogate for patient-centred outcomes (e.g. gout flares). It has not been clearly demonstrated that the strength of the relationship between SU and patient-centred outcomes is strong enough for SU to be considered a surrogate. 
Objectives: To determine the strength of the relationship between SU and the incidence of gout flares and thereby to determine whether $\mathrm{SU}$ is a valid surrogate endpoint according to the BSES3 framework.

Methods: A systematic search of Medline via PubMed, the Cochrane Central Register of Controlled Trials, EMBase via OVID, the Institute for Scientific Information Web of Science, and other sources identified relevant studies. Standardised data elements were extracted by 2 independent reviewers (LS, $\mathrm{MBM}$ ) and disagreements resolved by discussion (RC). Eligible trials were parallel-group, randomised trials of ULT of at least 3 months duration in people with gout. For the meta-regression analysis, a mixed linear model was used to combine the ratio of flare rates between groups (SAS software, v9.4 for Windows). Trials with multiple arms were treated as individual trials, referred to as "randomised comparisons" (i.e., 3 -arm trials with 2 active interventions was handled as 2 randomised comparisons). Data was analysed by meta-regression using the between-arm difference in proportion of individuals who achieved target $\mathrm{SU}(<0.36 \mathrm{mmol} / \mathrm{L})$ as independent variable from at 3 months (or 6 and 12 months if 3 month values not available) against flare rate (dependent variable).

Results: After screening 234 abstracts, 82 trials were scrutinized, of which 9 trials (with 16 comparisons) met inclusion criteria. A total of 5,696 people with gout entered into the meta-regression model. The longest RCT included was only 12 months duration. The pooled Odds Ratio (OR) suggested a small but statistically significant favourable association between the active and comparator urate lowering therapies and flare frequency (OR, $0.83 ; 95 \% \mathrm{Cl} 0.70$ to 0.99$]$ ). Substantial heterogeneity was present (between trial variance: $0.07 ; 0.03$ to 0.30 ). Meta-regression analysis did not reveal any statistically significant association between the proportion of individuals who achieved target SU and the observed flare rate $(P=0.82)$; the model fit did not improve after inclusion of the covariate into the model (between trial variance: $0.08 ; 0.03$ to 0.33 ) (Figure).

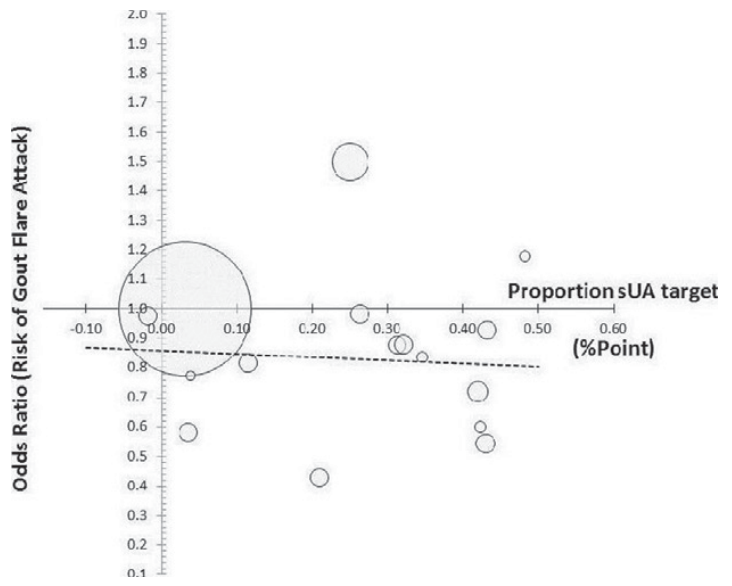

Conclusions: Substituting surrogate endpoints (proportion achieving target $\mathrm{SU}$ ) for the important clinical outcome (gout flares) allows conduct of shorter smaller trials. However, based on aggregate trial-level data (meta-regression) an anticipated association between SU and gout flare could not be confirmed. Trial duration may have been too short to observe a reduction in flares and further work using data from long-term extension studies is underway.

Disclosure of Interest: None declared

DOI: 10.1136/annrheumdis-2017-eular.1544

\section{OP0268 NURSE-LED CARE VERSUS GENERAL PRACTITIONER CARE OF PEOPLE WITH GOUT: A UK COMMUNITY-BASED RANDOMISED CONTROLLED TRIAL}

M. Doherty ${ }^{1}$, W. Jenkins ${ }^{1}$, H. Richardson ${ }^{1}$, A. Abhishek ${ }^{1}$, D. Ashton ${ }^{1}$, C. Barclay ${ }^{1}$, L. Duley ${ }^{2}$, H. Jones ${ }^{1}$, M. Santarelli ${ }^{1}$, A. Sarmanova ${ }^{1}$, M. Stevenson ${ }^{3}$, W. Zhang ${ }^{1}{ }^{1}$ Division of Rheumatology, Orthopedics and Dermatology; ${ }^{2}$ Clinical Trials Unit, University of Nottingham, Nottingham; ${ }^{3}$ Health Economics and Decision Science, University of Sheffield, Sheffield, United Kingdom

Background: Despite increasing prevalence of gout in the UK (1), a variety of barriers result in suboptimal care $(1,2)$ with only $40 \%$ of gout patients receiving urate-lowering therapy (ULT), usually at fixed dose without titration to a serum uric acid (SUA) target $(1,2)$. Nurses successfully manage many chronic diseases in the community, and we have shown that when people with gout are fully informed and involved in management decisions uptake of ULT is high and subsequent adherence under nurse-led care is excellent (3).

Objectives: To directly compare nurse-led care to general practitioner (GP) care of people with gout in a 2 year randomised controlled trial (NIHR CRN Portfolio No.12943)

Methods: 517 participants with acute gout in the previous year were identified from 56 local GP practices and randomised to nurse-led or continuing GP care. The nurses were trained about gout and its management according to recommended best practice (EULAR and BSR guidelines) involving full information, addressing illness perceptions, and involving patients in management decisions. Assessments were undertaken at 1 and 2 years. Analysis was intention to treat (last observation carried forward).

Results: Nurse $(n=255)$ and GP ( $n=262)$ groups were well matched at baseline for mean age (62 v 64yrs), sex ( $90 \% \vee 89 \%$ men), mean disease duration $(11.6$ $v 12.7 \mathrm{yrs})$, mean gout attack frequency in prior year (4.2 v. 3.8), tophi $(13.7 \%$ v. $8.8 \%)$, mean SUA ( 443 v. $439 \mu \mathrm{mol} / \mathrm{L})$, mean eGFR (71.5 v. 70.2) and ULT use $(40 \%$ v. $39 \%)$ (all $p>0.05)$. By 2yrs, $22(8.6 \%)$ and $54(20.6 \%)$ participants had discontinued the nurse and GP groups $(p<0.001)$, including 2 v. 8 deaths respectively. Comparing nurse and GP groups at 2 yrs: $95 \%$ v. $29 \%$ had SUA $<360 \mu \mathrm{mol} / \mathrm{L}$ (primary outcome); $88 \%$ v. $16 \%$ had SUA $<300 \mu \mathrm{mol} / \mathrm{L}$; mean (SD) SUA was $252 \pm 73$ v. $418 \pm 106 ; 97 \%$ v. $54 \%$ were on ULT; and mean (SD) dose of allopurinol was 470 (140) v. 240 (107) mg/day (all $p<0.001)$. Mean (SD) attack frequency during the 2nd year was $0.33(0.93)$ in the nurse v. $0.94(2.03)$ in the GP group $(p<0.001)$, and at $2 y$ rs tophi were present in $2.6 \%$ (reduced) $v$. $9.6 \%$ respectively $(p<002)$. Although equivalent at baseline, mean (SD) SF-36 norm-based physical component scores were better at 2yrs in the nurse group (41.31 (16.76) v. 37.87 (14.31); $p<0.05)$.

Conclusions: Nurse-led care of people with gout in the UK community can result in high uptake and excellent adherence to ULT over a 2yr period, achievement of target SUA in $>9 / 10$ cases and consequent improvements in patient-centred outcomes and quality of life. This study reinforces the benefits of "treat-to-target". Compared to standard GP care this model is likely to be cost effective long-term and merits further consideration.

References:

[1] Doherty M. et al. Ann Rheum Dis 2012;71:1765-70.

[2] Kuo C-F. et al. Ann Rheum Dis 2015;74:661-7.

[3] Rees F. et al. Ann Rheum Dis 2013:72:826-30.

Acknowledgements: Arthritis Research UK (Award No.19703) funded this study.

Disclosure of Interest: M. Doherty Grant/research support from: AstraZeneca, Consultant for: AstraZeneca, Grunenthal, Mallinckrodt and Roche, W. Jenkins: None declared, H. Richardson: None declared, A. Abhishek Grant/research support from: AstraZeneca, D. Ashton: None declared, C. Barclay: None declared, L. Duley: None declared, H. Jones: None declared, M. Santarelli: None declared, A. Sarmanova: None declared, M. Stevenson: None declared, W. Zhang Consultant for: AstraZeneca and Grunenthal DOI: 10.1136/annrheumdis-2017-eular.5006

\section{OP0269 EFFECT OF XANTHINE OXIDASE INHIBITORS ON THE INCIDENCE OF CARDIOVASCULAR EVENTS: A SYSTEMATIC REVIEW AND META-ANALYSIS OF RANDOMISED CONTROLLED TRIALS}

M. Bredemeier, L.M. Lopes, M.A. Eisenreich, G.K. Bongiorno, S. Hickmann. Rheumatology Division, Hospital Nossa Senhora da Conceição - Grupo Hospitalar Conceição, Porto Alegre - RS, Brazil

Background: There is evidence that xanthine oxidase inhibitors (XOI) may reduce the risk of major adverse cardiovascular events (MACE) $(1,2)$ and lower blood pressure (3). To date, this evidence is based mainly on observational studies (2). Objectives: To compare the incidence of MACE, mortality, and total and specific cardiovascular $(\mathrm{CV})$ events in patients enrolled in randomized controlled trials (RCTs) comparing XOI with placebo or no treatment.

Methods: A systematic review (CRD42015016073) searching for RCTs using PubMed, EMBASE, Cochrane Library, Web of Science, and Lilacs databases, and hand searching, was ended in Dec 2016. All RCTs comparing XOls with placebo or no treatment lasting $\geq 4$ weeks and including only adult individuals were eligible. The primary outcomes were the incidence of MACE (CV death, non-fatal myocardial infarction, unstable angina requiring urgent revascularization, or non-fatal stroke) and mortality; total CV events (TCE), specific CV outcomes, and serious adverse events (SAE) served as secondary outcomes. Associations were tested using the Peto odds ratio $(\mathrm{OR})$ without zero-cell continuity correction.

Results: In total, 81 studies including approx, 11,000 individuals reported extractable data on $\mathrm{CV}$ events. The use of $\mathrm{XOI}$ tended to be associated with lower incidence of MACE (OR=0.64, 95\% Cl 0.41-1.01, $\mathrm{P}=0.056)$, but not with mortality $(0.95,0.63-1.44)$. However, there was a significantly reduced incidence of TCE $(0.66,0.54-0.80, P<0.001)$, especially new/worsening hypertension $(0.57$, $0.37-0.87, P=0.009$ ), and a trend for reduction in the incidence of new/worsening heart failure $(0.74,0.53-1.04, P=0.086)$. The incidence of SAE $(0.86,0.71-1.05)$ did not differ significantly. Subgroup analysis suggested a protective effect for MACE in studies with high prevalence $(>50 \%)$ of cardiac diseases $(0.52,0.30-$ $0.91, P=0.021$ ). Sensitivity analysis excluding studies at high or unknown risk of bias produced no significant change in results, but reinforced the association with reduced incidence of hypertension $(0.26,0.11-0.60, \mathrm{P}=0.001)$ and heart failure $(0.55,0.32-0.94, P=0.030)$. Separate analysis of data on purine-like $\mathrm{XOI}$ (allopurinol and oxypurinol) confirmed the results of the primary analysis. Exploratory metaregression analysis showed association of dose of allopurinol with higher incidence of TCE $(P=0.023$, random effects $)$ and SAE $(P<0.001$, see Figure 1). Accordingly, in the subgroup with doses $\leq 300 \mathrm{mg} /$ day of allopurinol, a reduction of incidence of MACE $(0.36,0.18-0.68, P=0.002)$, TCE $(0.38,0.26-$ $0.54, \mathrm{P}<0.001)$, and $\mathrm{SAE}(0.49,0.34-0.71, \mathrm{P}<0.001)$ was observed, while the $\mathrm{SAE}$ risk increased in doses $>300 \mathrm{mg} /$ day $(1.39,1.04-1.91, P=0.047)$. There was 